UCRL-JC-123911

PREPRINT

\title{
Quantitative High Resolution Electron Microscopy of Grain Boundaries
}

\author{
Geoffrey H. Campbell \\ Wayne E. King \\ Dov Cohen \\ C. Barry Carter
}

This paper was prepared for submittal to the

MRS Fall Meeting, Boston, MA

December 2-6, 1996

December 12, 1996

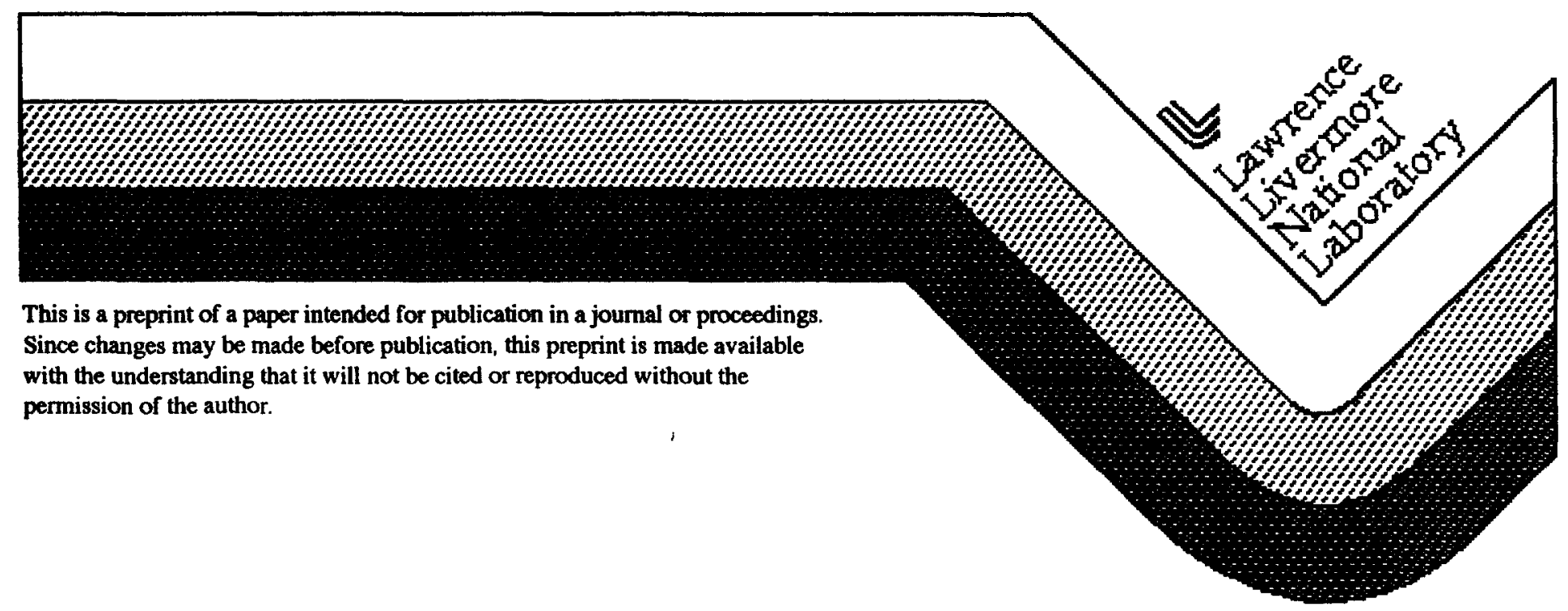




\section{DISCLAIMER}

This document was prepared as an account of work sponsored by an agency of the United States Government. Neither the United States Government nor the University of California nor any of their employees, makes any warranty, express or implied, or assumes any legal liability or responsibility for the accuracy, completeness, or usefulness of any information, apparatus, product, or process

disclosed, or represents that its use would not infringe privately owned rights. Reference herein to any specific commercial product, process, or service by trade name, trademark, manufacturer, or otherwise, does not necessarily constitute or imply its endorsement, recommendation, or favoring by the United States Government or the University of California. The views and opinions of authors expressed herein do not necessarily state or reflect those of the United States Government or the University of California, and shall not be used for advertising or product endorsement purposes. 


\title{
QUANTITATIVE HIGH RESOLUTION ELECTRON MICROSCOPY OF GRAIN BOUNDARIES
}

\author{
GEOFFREY H. CAMPBELL,* WAYNE E. KING,* DOV COHEN,** AND C. BARRY CARTER** \\ * University of California, Lawrence Livermore National Laboratory, Chemistry and Materials \\ Science Directorate, P.O. Box 808, Livermore, CA 94550 \\ ** University of Minnesota, Department of Chemical Engineering and Materials Science, Minneapo- \\ lis, MN, 55455
}

\section{ABSTRACT}

The $\sum 11(113) /[1 \mathrm{~T} 0]$ symmetric tilt grain boundary has been characterized by high resolution transmission electron microscopy. The method by which the images are prepared for analysis is described. The statistics of the image data have been found to follow a normal distribution. The electron-optical imaging parameters used to acquire the image have been determined by non-linear least-square image simulation optimization within the perfect crystal region of the micrograph. A similar image simulation optimization procedure is used to determine the atom positions which provide the best match between the experimental image and the image simulation.

\section{INTRODUCTION}

The atomic structure of lattice defects in the face-centered cubic (FCC) metals can be effectively modeled with atomistic potentials based on the Embedded Atom Method (EAM). However, for some of these metals, such as aluminum, the EAM potentials cannot be fit to all the available material parameters simultaneously. Thus, differing potentials are available which have been optimized to reproduce different aspects of the metal, such as lattice parameter, phonon dispersion curves, or point defect formation energy. It is not immediately clear which material parameters are appropriately optimized if the goal is to model the atomic structure of grain boundaries.

The $\Sigma 11(113) /[1 \overline{1} 0]$ symmetric tilt grain boundary in aluminum has been found to have a good qualitative match to predictions of its structure by atomistic simulations [1]. We report here preliminary results of an investigation of its atomic structure by quantitative high resolution electron microscopy (HREM). Although only a single EAM potential has been compared to the experimental results, slight differences have been found between the predicted and the observed structures.

\section{METHODS}

Model grain boundaries have been fabricated by ultra-high vacuum diffusion bonding [2] of single crystals. High resolution images of the boundary structure were acquired on a JEOL JEM-4000EX. The methods of data acquisition and handling are described in more detail elsewhere [3], as are the image simulation optimization procedures $[4,5]$. The main points are described here briefly.

\section{Data acquisition and digitization}

The method of quantifying a HREM image requires that certain steps be taken during the operation of the microscope. It must be realized that the image simulations produce an image represented in units relative to the incident electron intensity $\left(\mathrm{I}_{0}\right)$. Thus, the images acquired at the microscope must be normalized by $I_{0}$ in order to compare them quantitatively with the simulations. The easiest way to achieve this goal is to keep the electron dose (intensity multiplied by time) constant for each image in a through focal series by maintaining a constant beam convergence and exposure time. After acquiring the through focal series, the incident intensity is measure by removing the specimen from the electron beam and recording an image under the same conditions. When the HREM images are normalized by the image of the incident beam, the result is in the same units as the image simulation.

The images are acquired on film due to its ease of use and large area of detection. The images are digitized by placing the processed negative on a spherical-illumination light table and using a CCD camera with a short focal length telescope to measure the optical transmittance (fraction of light transmitted) as a function of position in the area of interest. The magnification of the image is chosen such that the pixel-to-pixel spacing is close to that produced by the HREM image simulation. The darkening of 


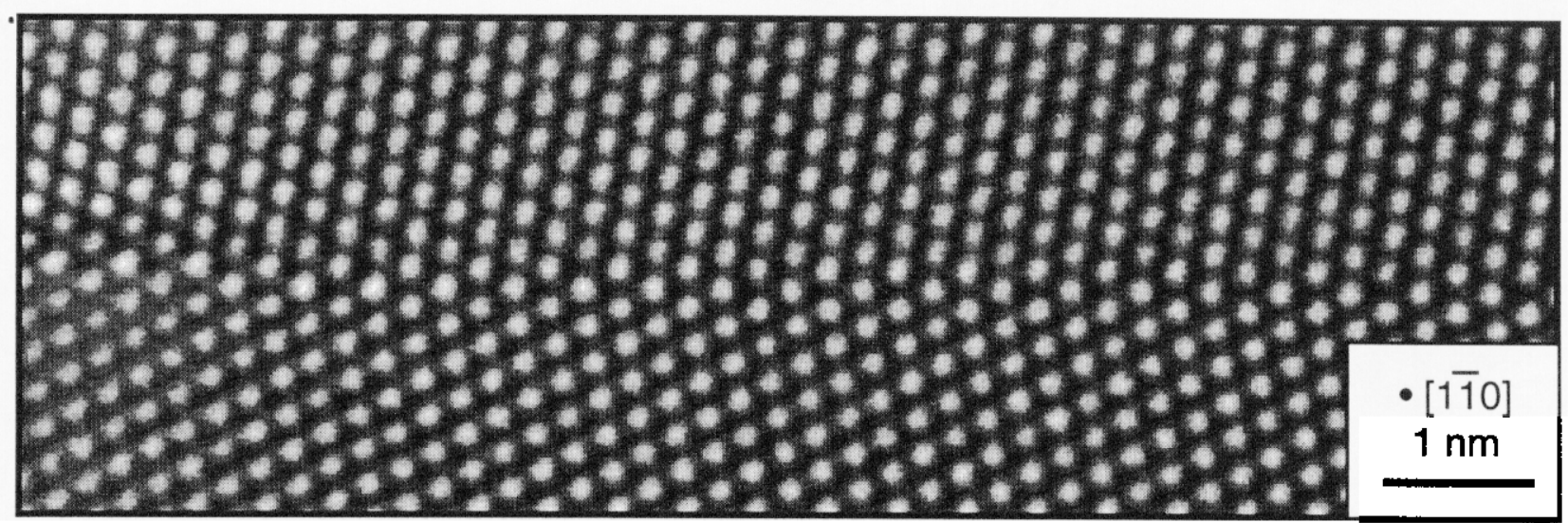

Figure 1: A high resolution electron micrograph of the $\sum 11$ (113)/[110] symmetric tilt grain boundary in aluminum. This micrograph provided the experimental data for the analysis of the grain boundary structure.

film is not a linear function of electron dose. Thus, a calibration must be performed to relate the optical transmittance of the film to the relative electron dose. This calibration is performed on both the HREM image and the image of the incident beam before the ratio is taken to yield the final image in the correct units.

\section{Distortion correction and average image formation}

In the subsequent sections, it will be seen that the analysis of the grain boundary structure is a two step process. The first step is to examine the regions of perfect crystal, away from the boundary, where the positions of the atoms are known, to determine the microscope imaging parameters. The second step is to examine the grain boundary structure, where the atom positions are not necessarily known, but now with the knowledge of the imaging parameters. Therefore, it is necessary to examine data from these two regions of the micrograph separately.

The analysis proceeds by forming an average image of the contrast in the perfect crystal. Associated with each pixel in the average image will be a standard deviation to describe the scatter of the original data about the average value. We refer to this as the standard deviation image. In forming the average image, some of the electron optical distortions present in the image can be removed by imposing the known rectangular symmetry on the image. Careful alignment of the average image with the image simulation is also necessary. The average and standard deviation images are also formed for the repeat unit of the grain boundary.

\section{Image simulation optimization}

The average image of the perfect crystal is compared quantitatively to the high resolution image simulation and the simulation is optimized by a non-linear, least-squares optimization routine. The figure of merit in the optimization is called the normalized residuals, $R$, and is defined as

$$
R_{i}(x)=\frac{I_{i}^{\exp }-\left(I_{i}^{\text {calc }}(x)+b^{f i t}\right)}{\sqrt{\left(\sigma_{i}^{\exp } / \sqrt{N}\right)^{2}+\left(\sigma_{c a l}^{\exp }\right)^{2}}}
$$

where the evaluation is at each pixel, $\mathbf{i}$, and is a function of the electron optical imaging parameters, $\mathbf{x}$, used to calculate the simulated high resolution image, $I^{\text {calc }}$. The numerator of the expression is the difference between the experimental average image, $\mathrm{I}^{\mathrm{exp}}$, and the simulated image with a fitted constant background term, $\mathrm{b}^{\mathrm{fit}}$. The denominator is the estimation of the experimental error. The standard deviation of the data at each pixel in the individual contrast units is given by $\sigma^{\exp }, \mathrm{N}$ is the number of contrast units over which the average is formed, and the second term, $\sigma_{c a l}^{\exp }$, is the standard deviation of the difference 


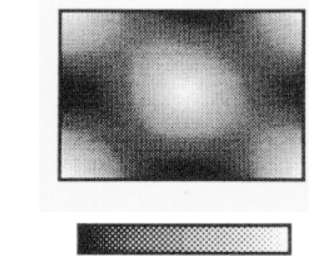

0.52

0.92
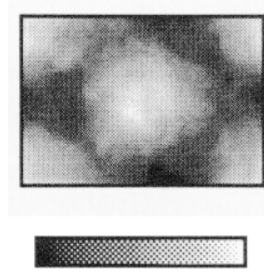

0.001

0.032

Figure 2: Average image and standard deviation image of perfect crystal, with intensity scales.

acterization by HREM straightforward.

of the measured points from the fitted curve in the film density to electron dose calibration. Both errors are independent and evaluated at the $67 \%$ confidence level, therefore they are added in quadrature [6]. The significance of the normalized residuals is that if they range between \pm 1 then the calculated image matches the experimental image to within the experimental error. The optimization routine proceeds by minimizing the sum of the squares of this quantity by varying the simulation inputs such as microscope focus, specimen thickness, beam tilt, etc. The high resolution images are simulated with EMS[7].

\section{Grain boundary structure refinement}

Once the imaging parameters for a given micrograph have been determined by examining the perfect crystal, these quantities can be held constant while the grain boundary is examined. The same type of optimization is performed, but now the only quantities allowed to vary are the positions of the atoms used to form the grain boundary structure in the image simulation.

\section{RESULTS}

A $\Sigma 11(113) /[1 \overline{1} 0]$ symmetric tilt grain boundary was fabricated by diffusion bonding and characterized by HREM, as shown in Figure 1. This grain boundary has a relatively low energy compared to other boundaries of nearby orientation, hence the boundary facets to the desired orientation over large areas. The exact orientation inherent in a grain boundary facet makes the char-

The average image and the standard deviation image for the perfect crystal are shown in Figure 2. A $12 \times 12$ grid of contrast units (144 total) were used to form the image. It is instructive to examine the variation of the individual data points about their average value. At each individual pixel in the average image, the difference from the average value of every data point used to form that average is tabulated. The intensity levels in the average image are arbitrarily divided into 10 bins. In Figure 3, these differences are plotted for two bins, each at either end of the intensity range. In each case, a Guassian curve has been fitted to the distribution and the peak width parameter, $\sigma$, is shown. When the area under the curve

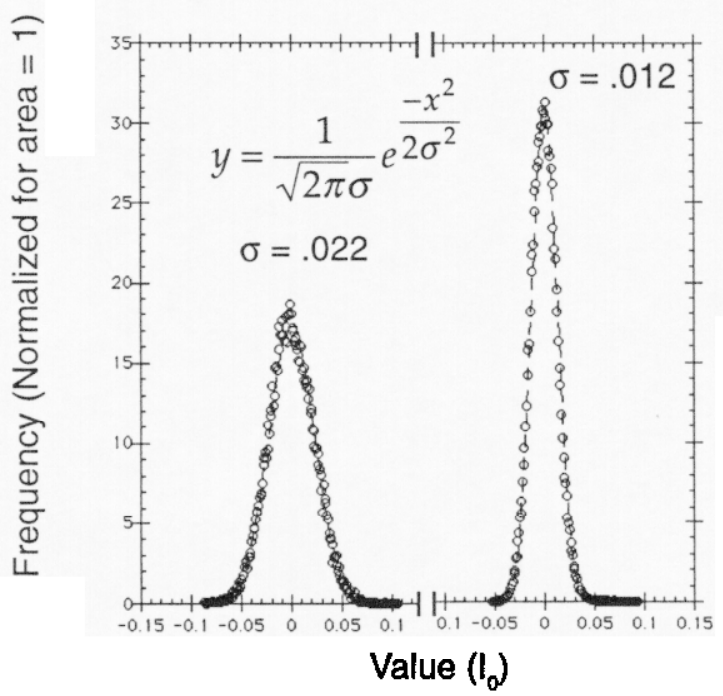

Figure 3: Two examples of the statistical distribution of the data points about their mean value for two windows of intensity (see Fig. 4). For each distribution, a Guassian curve as been fit to the data and the fitting parameter value is shown.

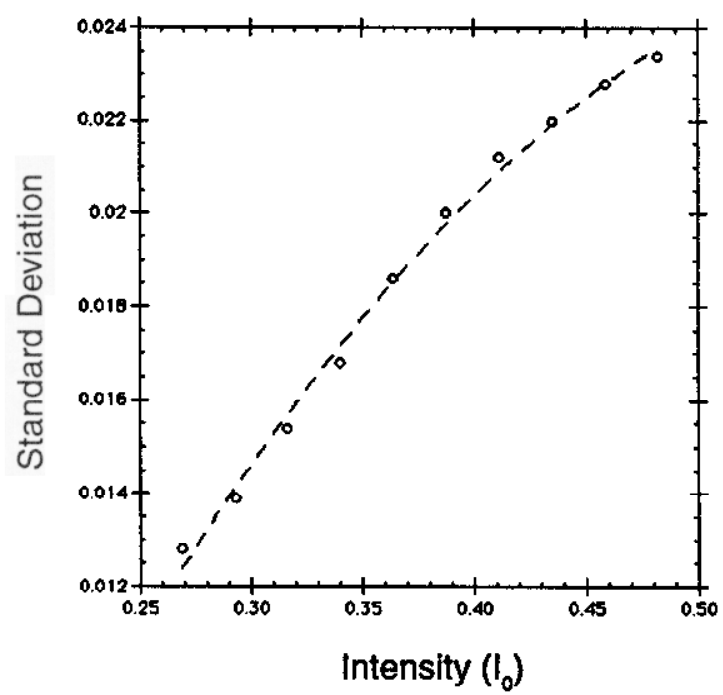

Figure 4: Plot of the fitting parameter from Fig. 3(the standard deviation) as a function of intensity. The fitted curve is a polynomial of order 2. 


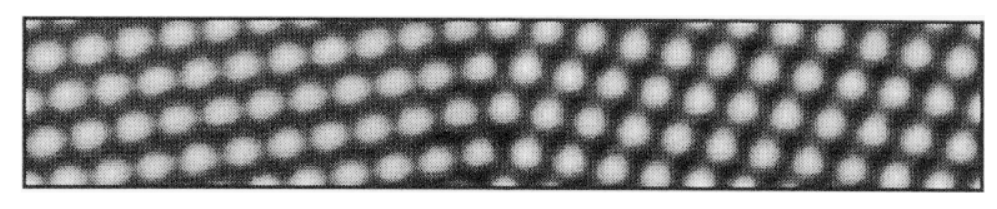

$0.52 \quad 0.92$

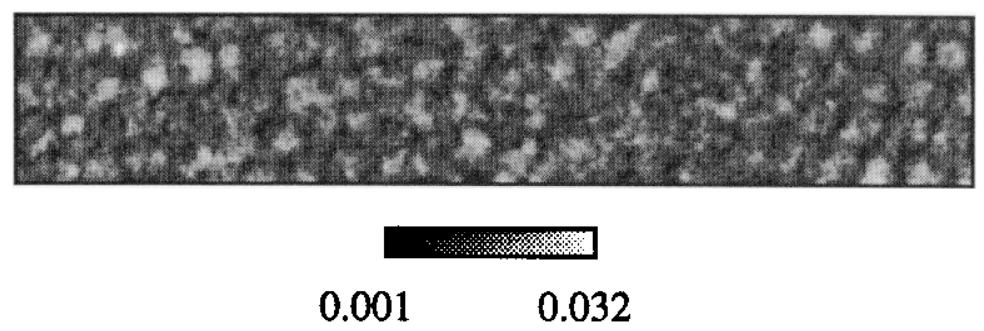

Figure 5: The average image and the standard deviation image of the structural unit of the grain boundary. The intensity scales are shown in units of fraction of incident intensity.

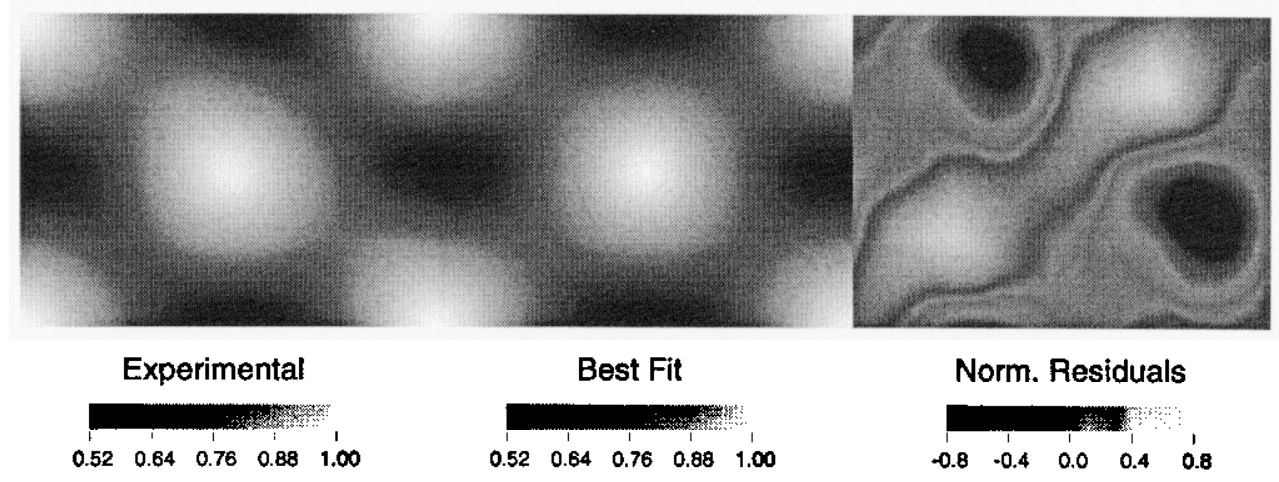

Figure 6: The average image of the unit cell of Al in the experimental micrograph is shown at the left. The center image the best fit image simulation of Al along $<110>$ which is a result of the optimization. The normalized residuals are shown at the right. An intensity scale accompanies each image.

is normalized to unity, then $\sigma$ is equal to the standard deviation of the data. The dependence of the standard deviation of the data on the intensity is shown in Figure 4. A line has been fitted to the data with a polynomial of order two. The average image and standard deviation image of the grain boundary are shown in Figure 5. A total of 6 images were used to form the average.

Image simulations of $\mathrm{Al}$ as viewed along [011] show that there is only one region in focus-thickness space that produces strong contrast. This behavior eliminates ambiguity caused by the possibility of finding local minima of the normalized residuals during the optimization. Although the rate of convergence during the optimization is dependent on the choice of initial conditions, it is found that the optimized image simulation does occur for a unique set of parameters (the " $x$ " in Equation (1)). The optimized image simulation for the region of perfect crystal on either side of the boundary is shown in Figure 6. The image simulation parameters found for the best match were a specimen thickness of $5.2 \mathrm{~nm}$, defocus of $65 \mathrm{~nm}$, defocus spread of $10 \mathrm{~nm}$, vibration in the $\mathrm{x}$-direction of $.06 \mathrm{~nm}$ and y-direction of .05 $\mathrm{nm}$, and a constant background level of -0.055 .

These parameters were used to simulate an image of the grain boundary using atom coordinates provided by an EAM type calculation. This image is shown in Figure 7 along with the image of the normalized residuals. A region in the plane of the boundary is seen to have an area of increased residuals indicating a poor fit between simulation and experiment. The optimized image simulation for the grain boundary image is shown in Figure 8 . In this case the atom positions near the boundary were allowed to vary. A small shift in the position of atoms adjacent to the boundary in the direction parallel to the boundary are seen to reduce the normalized residuals and thus improve the fit between the simulation and the experimental image. 


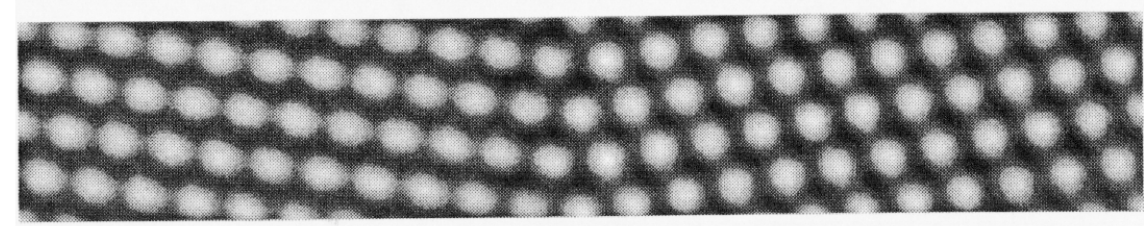

Experimental

$\begin{array}{lllll}0.52 & 0.62 & 0.72 & 0.82 & 0.92\end{array}$

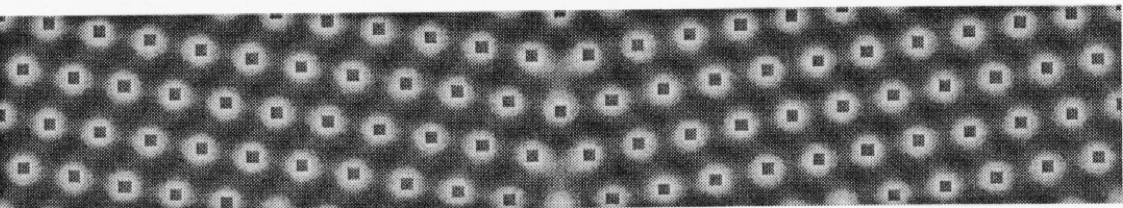

Simulation

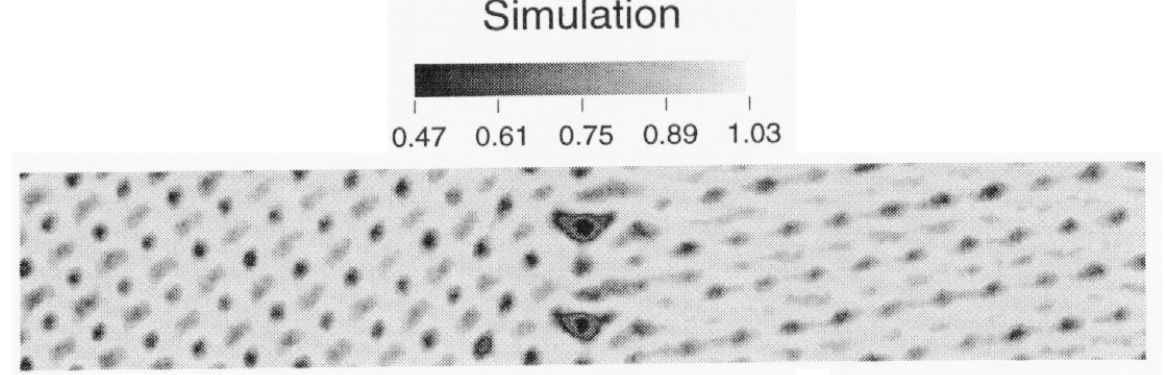

Residuals

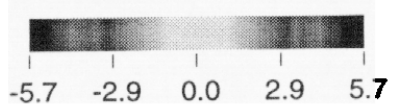

Figure 7: These images show the initial conditions used for the grain boundary structure refinement. The top image is the average image of the experimental micrograph of the grain boundary. The center image is the image simulation based on the prediction of an EAM calculation. The gray squares mark the atom positions. The bottom image is the normalized residuals. Note the high values of the residuals in the plane of the grain boundary.

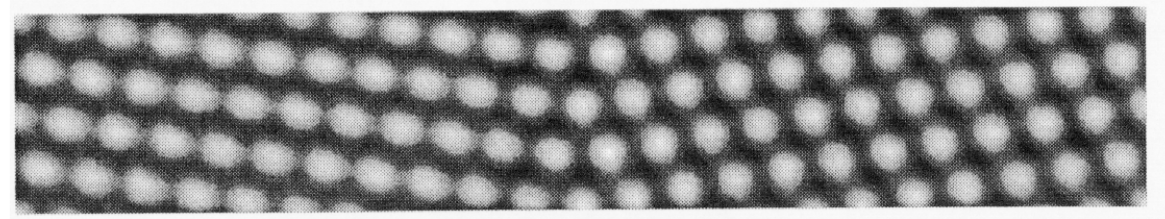

Experimental

$\begin{array}{lllll}0.52 & 0.62 & 0.72 & 0.82 & 0.92\end{array}$

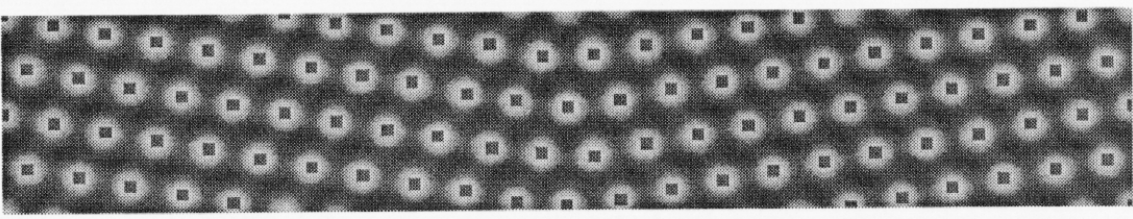

Simulation
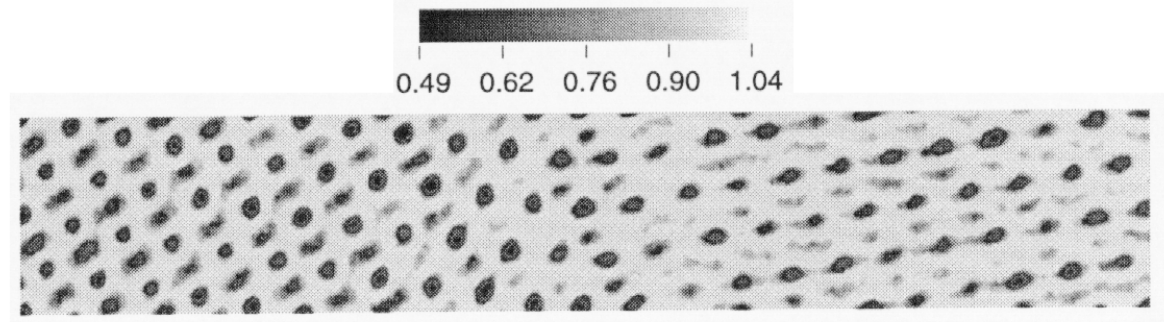

Residuals

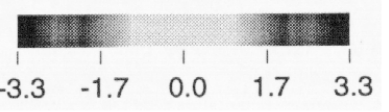

Figure 8: These images show the final configuration which is a result of the structure refinement. The top image is the same as in Fig. 7. The center image is the optimized image simulation. Comparison of the positions of the gray squares show slight shifts with respect to those in Fig. 7. The bottom image of the normalized residuals shows a reduction in their magnitude in the grain boundary. Not the change of intensity scale relative to Fig. 7. 


\section{DISCUSSION}

The analysis of the statistical distribution of the data in a high resolution image provides important information for their analysis. For example, in the examination of a contrast feature that does not have periodicity to exploit for averaging, such as a dislocation or a void, a straightforward inspection of their contrast levels with error bars will establish to within a well defined confidence level whether or not a change from one contrast feature to another is significant. In the case of grain boundaries, where periodicity allows averaging, it can be established how many contrast units are required to reduce the error to a point where features can be meaningfully distinguished. The curve fitting in Fig. 4 reveals that the counting statistics, which should have a dependence of order 2 , is not the only source of scatter.

Although an improved fit between the simulated image and the experimental image was found for slightly shifted atoms as compared to the predictions of the EAM model, the results are not yet conclusive. The contrast of the perfect crystal on either side of the grain boundary is slightly different. There are several possibilities for the origin of the difference, including specimen tilt, drift, two- and three-fold astigmatism. The effects of these parameters has not been fully explored in the image optimization due to the time intensive nature of the calculation. Also, only one model of the grain boundary structure has been compared to the experimental images. Other potentials based on the EAM may provide predictions more closely matching the observations. The differences in the normalized residuals for the perfect crystal regions in the simulation of the grain boundary as compared to the previous optimization for the perfect crystal reveal a difference in the conditions under which the two images were digitized. A procedure has been determined to eliminate this difference in the future.

\section{CONCLUSIONS}

A technique for the quantitative evaluation of high resolution electron micrographs has been developed. Its application to a $\Sigma 11(113) /[1 \overline{1} 0]$ symmetric tilt grain boundary has been demonstrated. The units of intensity in the experimental image have been converted to the same units as the simulated images. Analysis of the statistics of the experimental data show that they follow a normal distribution. The electron optical imaging parameters used to acquire the images can be determined from optimizing the match of the simulated image with the experimental image in the perfect crystal regions. The knowledge of the imaging parameters then allows the examination of the grain boundary to obtain its atomic structure through a similar image simulation optimization.

\section{ACKNOWLEDGMENTS}

We would like to thank Stephen Foiles for providing the EAM model structure. We would like thank Doug Medlin for the use of the $4000 \mathrm{EX}$ at Sandia National Lab, Livermore. DC would like to thank both Associated Western Universities and the NSF for partial support of this work under contract number NSF/DMR-9522253. This work performed under the auspices of the United States Department of Energy, Office of Basic Energy Sciences and Lawrence Livermore National Laboratory under Contract No. W-7405-Eng-48.

\section{REFERENCES}

1. M. J. Mills, M. S. Daw, G. J. Thomas and F. Cosandy, Ultramicroscopy, 40, p. 247 - 257 (1992).

2. W. E. King, G. H. Campbell, A. W. Coombs, G. W. Johnson, B. E. Kelly, T. C. Reitz, S. L. Stoner, W. L. Wien and D. M. Wilson in Joining and Adhesion of Advanced Inorganic Materials, edited by A. H. Carim, D. S. Schwartz and R. S. Silberglitt (Mat. Res. Soc. Symp. Proc. 314, Materials Research Society, Pittsburgh, PA 1993) pp. 61-67.

3. G. H. Campbell, D. Cohen and W. E. King, J. Microsc. Soc. Am., submitted (1996).

4. W. E. King and G. H. Campbell, Ultramicroscopy, 51, p. 128 - 135 (1993).

5. W. E. King and G. H. Campbell, Ultramicroscopy, 56, p. 46 - 53 (1994).

6. S. Rabinovich, Measurement Errors: Theory and Practice, American Institute of Physics, New York, NY, 1993, pp. 126.

7. P. A. Stadelmann, Ultramicroscopy, 21, p. 131 - 146 (1987). 


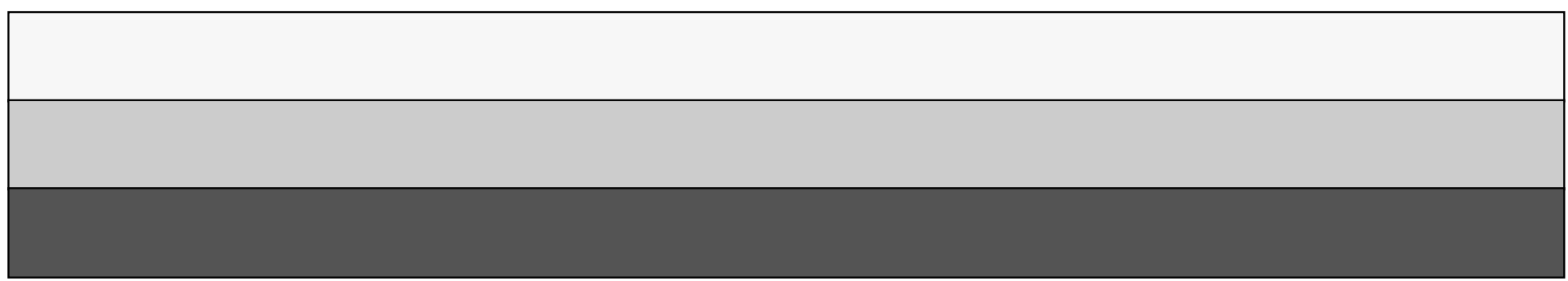

